\title{
Contribution à la connaissance de Colletes halophilus Verhoeff (Hymenoptera, Colletidae) en France et confirmation de sa présence en Aquitaine
}

\author{
Par David GENOUD * et François DITTLO **
}

\section{Introduction}

Le renouveau de l'Apidologie en France, la dynamique nouvelle créée et la méconnaissance de notre faune d'Apoïdes nous offre un immense terrain d'aventure et de découvertes. Un nouvel exemple nous en apporte la preuve. Suite à un échange de discussion sur le sujet des Colletidae estivaux du groupe de Colletes succinctus entre plusieurs membres de la liste de discussion informelle Apoidea-Gallica j'avais établi un plan de prospection pour la région Aquitaine (France) de l'espèce Colletes halophilus Verhoeff basé sur les foyers de présence de sa plante de prédilection: l'Aster maritime, Aster tripolium (Asteraceae) (Figure 1).

Cette espèce d'abeille oligolectique est bien connue de sites plus septentrionaux (E/S-E des côtes Anglaises, côtes Belges de la Manche et de la Mer du Nord, côtes Néerlandaises). Sa présence en France n'a été confirmée que récemment et les dernières prospections établies signalent l'espèce dans l'Ile et Vilaine (Saint-Malo) et le Finistère (comm. pers. Stuart Roberts \& Gilles Mahé 2006). Colletes halophilus était donc à rechercher en Aquitaine sur les sites favorables.

\section{Sites potentiels en Aquitaine}

- Sites potentiels dans les Landes (F-40) : du Sud au Nord sur les communes de Tarnos (Estuaire et zone amont) et Saint-Martin-de Seignanx en rive droite de l'Adour, Capbreton, Hossegor (Lac d'Hossegor), Vieux-Boucau (Lac marin), Vielle Saint-Girons (Courant d'Huchet) puis tous les grands lacs au Nord de Mimizan;

- Sites potentiels dans les Pyrénées-Atlantiques (F-64): du Nord au Sud sur les communes d'Anglet, de Bayonne et de Mouguerre en rive gauche et droite de l'Adour et sur la basse Nive, du côté de Bidart (basse Uhabia), dans les environs de Saint-Jean-de-Luz (Basse Nivelle) et à Hendaye (Bidassoa aval). Les stations d'Aster tripolium sont peu nombreuses et très disséminées, elle est protégée au niveau départemental dans le 64;

* Rue de Conseillé 29bis, F-40220 Tarnos, France.

E-mail: antidge@aol.com

** La Garenne 3, F-3373 Villandraut, France.

E-mail: fdttl@yahoo.fr

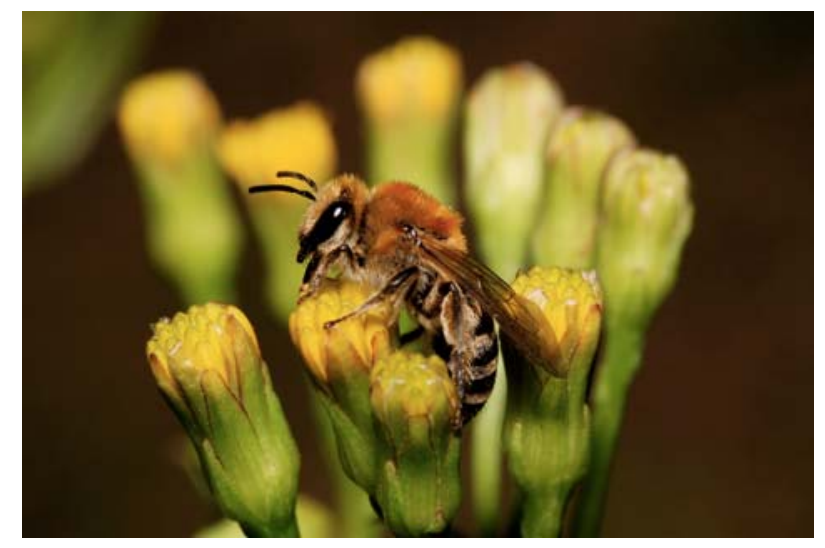

Figure 1. Femelle de Colletes halophilus (Hym. Colletidae) sur une inflorescence d'Aster tripolium (Asteraceae) (Photo N. Vereecken)

- Sites potentiels en Gironde (F-33) : sur l'ensemble de l'Estuaire de la Gironde en se raréfiant à partir du Bec d'Ambès mais jusqu'à Langoiran sur la Garonne. L'ensemble du contexte des étangs côtiers médocains et du Bassin d'Arcachon en contact avec des milieux salins.

\section{Ces sites sont illustrés à la Figure 2.}

Le 7 septembre 2006, j’ai reçu par la poste une paire d'exemplaires de Colletes récoltées à Lanton sur le Bassin d'Arcachon par François Dittlo. J'ai étudié ces exemplaires le 9 septembre et j'ai pu en conclure que nous avions bien affaire à Colletes halophilus. Le 11 septembre, je suis parti prospecter la rive droite de l'Adour sur les communes de Tarnos (F-40), Boucau et Bayonne (F-64). Aucune présence de Colletes halophilus n'est décelée sur différents sites (3 sites prospectés) où la présence au stade de floraison de la plante de prédilection est aléatoire du fait d'un fauchage régulier des berges en site urbain. J'ai ensuite effectué une visite 30 kilomètres au Nord sur le Lac marin d'Hossegor (F-40). Ce lac en univers urbain présente en rive droite des faciès de vase exondée dominés par l'Aster maritime, Aster tripolium (Figure 3). Après une courte recherche j'ai capturé, sur la partie centrale, 3 mâles de Colletes halophilus. Ceux-ci n'hésitant pas à venir bruyamment patrouiller autour de moi ${ }^{1}$. 


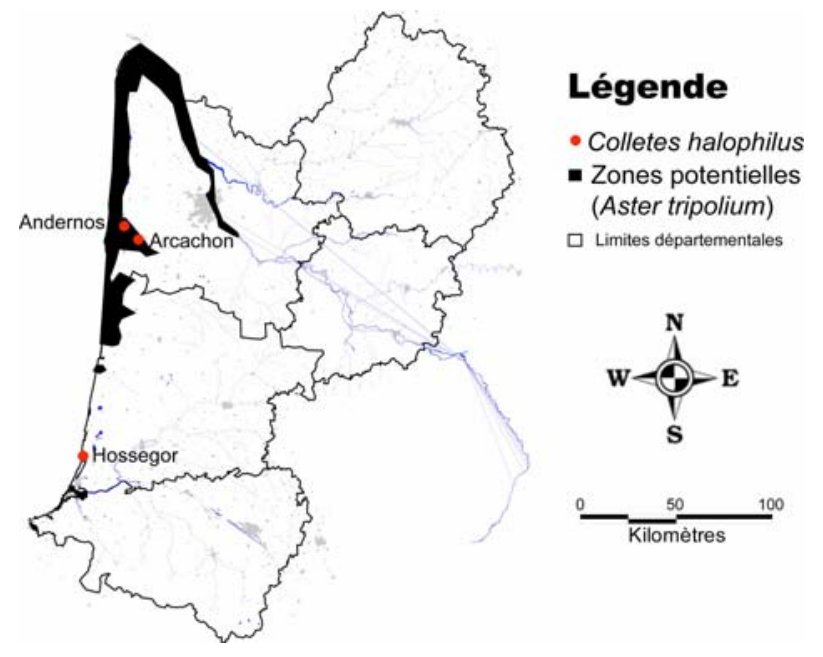

Figure 2. Sites potentiels en Aquitaine (SO-France) pour Colletes halophilus (Hym. Colletidae) (Illustration D. Genoud)

Le 12 septembre, Stuart Roberts (Université de Reading, Angleterre) m'informe de la confirmation par George Else (Museum National d'Histoire Naturelle de Londres, Angleterre) de la présence de Colletes halophilus à Lanton sur le Bassin d'Arcachon (F-33) sur la base d'individus transmis par François Dittlo. Le 29 septembre, l'identité des individus prélevés au Lac d'Hossegor (F-40) a été confirmée par Stuart Roberts et Nicolas Vereecken.

\section{Conclusion et perspectives}

Cette grande avancée géographique de la connaissance de l'aire de répartition de Colletes halophilus augure de sa présence sur l'ensemble de la côte Atlantique française, les côtes du Morbihan, de la Vendée et de la Charente-maritime présentent nombre de stations à Aster maritime.

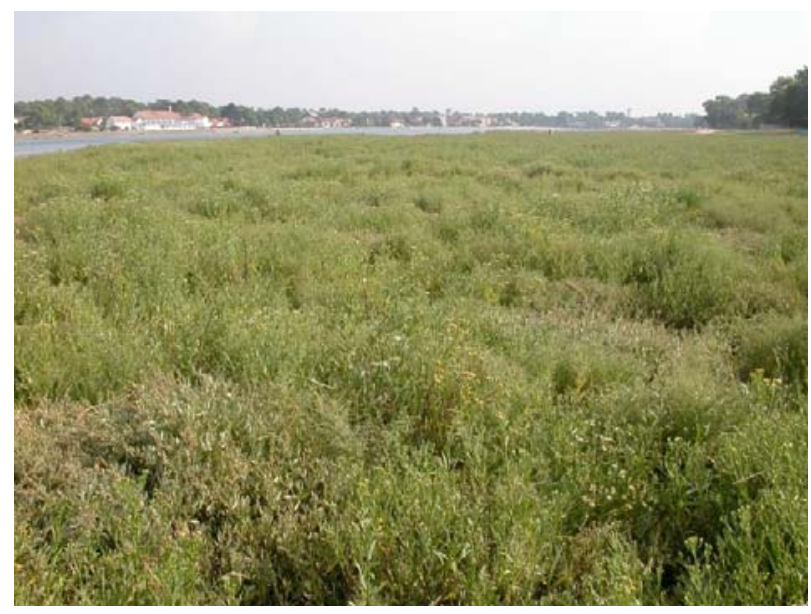

Figure 3. Aperçu du site du lac d'Hossegor (F-40), dominé par Aster tripolium (Asteraceae) (Photo D. Genoud)

L'aire de répartition de l'espèce pourrait nous amener à découvrir l'espèce bien au-delà de nos frontières sur les côtes basques espagnoles mais aussi plus au Sud en Guipuzcoa ou en Galice. Se pose déjà la question de la présence de l'espèce à de telles latitudes : est-ce le fruit d'une extension plus ou moins récente ou de l'absence de prospection visant cette espèce auparavant?

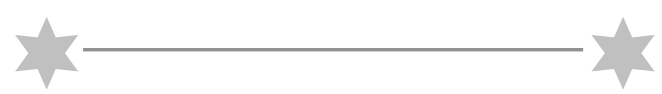

